Check for updates

Cite this: RSC Adv., 2019, 9, 3857

Received 2nd November 2018

Accepted 22nd January 2019

DOI: 10.1039/c8ra09093a

rsc.li/rsc-advances

\section{Redox chemistry in the pigment eumelanin as a function of temperature using broadband dielectric spectroscopy $\dagger$}

\begin{abstract}
K. A. Motovilov, (D) *a V. Grinenko, ${ }^{\text {bc }}$ M. Savinov, ${ }^{d}$ Z. V. Gagkaeva, ${ }^{a}$ L. S. Kadyrov, ${ }^{a}$ A. A. Pronin, ${ }^{e}$ Z. V. Bedran, ${ }^{a}$ E. S. Zhukova, ${ }^{a e}$ A. B. Mostert (iD ${ }^{* f}$ and B. P. Gorshunov ${ }^{a e}$

Conductive biomolecular systems are investigated for their promise of new technologies. One biomolecular material that has garnered interest for device applications is eumelanin. Its unusual properties have led to its incorporation in a wide set of platforms including transistor devices and batteries. Much of eumelanin's conductive properties are due to a solid state redox comproportionation reaction. However, most of the work that has been done to demonstrate the role of the redox chemistry in eumelanin has been via control of eumelanin's hydration content with scant attention given to temperature dependent behavior. Here we demonstrate for the first time consistency between hydration and temperature effects for the comproportionation conductivity model utilizing dielectric spectroscopy, heat capacity measurements, frequency scaling phenomena and recognizing that activation energies in the range of $\sim 0.5 \mathrm{eV}$ correspond to proton dissociation events. Our results demonstrate that biomolecular conductivity models should account for temperature and hydration effects coherently.
\end{abstract}

\section{Introduction}

Conductive biomaterial systems are actively being investigated for potential insights that may lead to technological applications. These biomaterial systems and their applications are quite diverse ${ }^{1-4}$ and include specific systems such as bacterial extracellular filaments, ${ }^{4-6}$ conductive protein mats ${ }^{7,8}$ and doped polysaccharides, ${ }^{9}$ to select a few. One such material that is receiving a lot of attention is the biomacromolecular system eumelanin as a model bioelectronic material. ${ }^{10}$ Eumelanin has already been tested in various devices platforms such as UV filters, ${ }^{\mathbf{1 1}}$ solid-state organic electrochemical transistors, ${ }^{\mathbf{1 2}}$ metalinsulator devices, ${ }^{13}$ flexible supercapacitors, ${ }^{14}$ extended gate field effect transistors, ${ }^{\mathbf{1 5}}$ engineered electrodes ${ }^{\mathbf{1 6}}$ and edible batteries. ${ }^{17}$

Eumelanin, the archetypal melanin and referred to as melanin from now on, is part of a family of melanins primarily associated with photo-protection and pigmentation. ${ }^{18-20}$ It is

\footnotetext{
${ }^{a}$ Moscow Institute of Physics and Technology, 141701, Institutsky lane 9, Dolgoprudny, Russia.E-mail: motovilov.ka@mipt.ru

${ }^{b}$ Institute for Solid State and Materials Physics, TU Dresden, 01069, Dresden, Germany 'Institute for Metallic Materials, IFW Dresden, 01069, Dresden, Germany

${ }^{d}$ Institute of Physics AS CR, Praha 8, Czech Republic

${ }^{e}$ Prokhorov General Physics Institute of the Russian Academy of Sciences, 119991, Vavilov Street 38, Moscow, Russia

${ }^{f}$ Department of Chemistry, Swansea University, Singleton Park, SA2 8PP, Wales, UK. E-mail: a.b.mostert@swansea.ac.uk

$\dagger$ Electronic supplementary information (ESI) available. See DOI: 10.1039/c8ra09093a
}

also found in areas of the body, such as in the substantia nigra of the brain stem, which would suggest other roles. This has led to speculation as to other functions such as radiation protection, free radicals scavenging and heavy metal ion chelation. ${ }^{18-23}$

An additional property is a free radical signal as measured by electron paramagnetic resonance (EPR)..$^{\mathbf{2 0 2 4 - 2 9}}$ The signal consists of two radicals: a carbon centered and semiquinone free radical. The most important for conductivity is the semiquinone radical (see below). ${ }^{26}$

The most relevant property for devices is melanin's hydration dependent conductivity. In the past five years it has been shown that melanin should be regarded, at minimum, as a mixed ionic/electronic conductor ${ }^{24,30,31}$ with a predominant proton component.

This hydration dependent effect is significant in that it can change the observed conductivity by orders of magnitude though the water content only increases linearly. ${ }^{24,30-33}$ Hence, it has become clear that any electrical study of melanin requires a degree of hydration control. The aforementioned mixed ionic/ electronic viewpoint should actually be further narrowed to viewing melanin as a proton conductor exclusively based upon recent impedance work in which thin films of melanin were subject to hydration control. ${ }^{34}$ Based upon this hydration dependent behaviour, the origin of the conductivity in melanin is ascribed to a comproportionation reaction (Fig. 1, pathways IV and V) where adding water yields hydronium and semiquinone radicals for conduction. As a result, what the recent hydration dependent work shows is that the previous four 
<smiles>[R]c1cc2c([nH]1)=CC(=O)C(=O)C=2</smiles><smiles>O=C(O)Cc1cc2cc(O)c(O)cc2[nH]1</smiles>

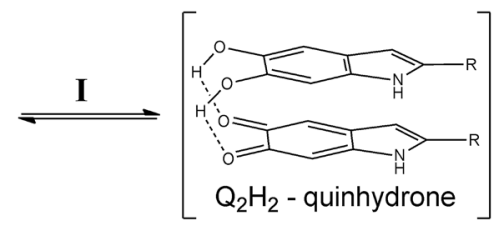

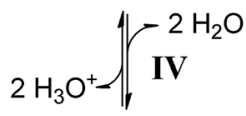<smiles>[R]OC(=O)C=I[Tl]</smiles><smiles>[R]c1cc2c([nH]1)=CC(=O)C(=O)C=2</smiles>

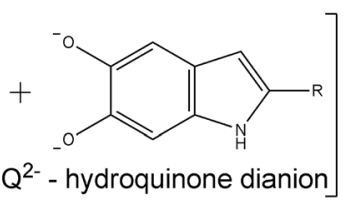<smiles>O=CC1=CC(=O)C(C(=O)O)=CC1=O</smiles>
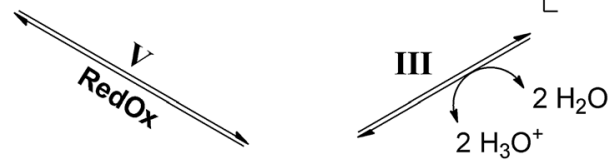<smiles>O=C1C=C2C=C(C(=O)O)C(=O)C=C2N1</smiles>

Fig. 1 Redox transformations of indolequinone blocks within melanin, which is built from two main monomer units, dihydroxyindole (DHI) and dihydroxyindole carboxylic acid (DHICA), both of which are hydroquinones. In the top left corner, a quinone (a different redox state of the hydroquinone) and hydroquinone are depicted. The $\mathrm{R}$ indicates either an $\mathrm{H}$ or $\mathrm{COOH}$ group commensurate with either an original $\mathrm{DHI}$ or $\mathrm{DHICA}$ starting monomer. Thus far most studies have understood the conductivity of melanin via the chain of equilibria entities labelled IV and V. In the dry state only equilibria I and II are possible. Addition of water shifts the system to formation of charged semiquinone anion form $\mathrm{SQ}^{-}$and generation of hydronium cations which play the main role in creating a pool of diffusible protons.

decades old amorphous semiconductor model for melanin ${ }^{20,35-37}$ should be discarded.

However, even with the recent progress, there is a noticeable absence in the recent melanin work on its conductivity behavior, which is a systematic investigation of its redox chemistry as a function of temperature. Most of the recent work has focused on hydration dependent behavior. Currently, this state of affairs needs to be rectified to confirm the validity of the proposed comproportionation reaction model, since it would be expected that there should be consistency between the hydration and temperature dependent conductivity behavior.

One way to study the conductivity, hence the redox chemistry of melanin, is by performing temperature dependent direct current (DC) and broad-band dielectric/alternating current (AC) measurements. Furthermore, information of molecular and charge dynamics is provided by specific heat measurements. However, when it comes to conductive biomaterials in general, one often finds that temperature dependent studies, either on wet or dry biomaterials, are done with no concern given to properly controlling the hydration content. ${ }^{\mathbf{1 0}}$ As has been demonstrated with the melanins, ${ }^{30}$ water content of a biomaterial is a first order variable for its conductive behavior. Other materials in which it is clear that water plays a significant role include the conductive bacterial filaments. ${ }^{38}$ Thus, doing a temperature dependent study without proper water content control will bring out confounding errors due to water evaporation or condensation into the sample. ${ }^{\mathbf{1 0}}$ Hence, the temperature dependent work presented here are on melanin samples in which the water content has been controlled.

To date there are only a few temperature dependent, conductivity studies that have been done on melanin, which is unusual given its promise. The most thorough and consistent temperature dependent AC and DC measurements, attempting to account for hydration, were undertaken by Jastrzebska et $a l .^{32,39,40}$ Their data indicated that the room temperature DC conductivity of melanin rises from $10^{-13}$ to $10^{-5} \mathrm{~S} \mathrm{~cm}^{-1}$ depending on the hydration level ${ }^{32}$ and found DC conductivity activation energies of $0.49-0.76 \mathrm{eV}$ depending on the level of vacuum and heating or cooling type of the measurement. The AC response of melanin as reported by Jastrzebska et al. ${ }^{\mathbf{4 0}}$ was measured in the frequency ranges of $\nu=10^{2}$ to $10^{9} \mathrm{~Hz}$ and $^{39}$ $10^{-2}$ to $10^{7} \mathrm{~Hz}$ with the latter study utilizing a broader temperature interval of 253-393 K. Critically, Jastrzebska et al. 's AC studies were performed without thorough control of ambient humidity. In all their analyses, they presumed a semiconductor model, which has proven to be untenable. Thus, the interpretation of their results is now open to question.

As to specific heat measurements, there exist only few such experiments on melanin, which include work on only a narrow temperature interval of $0.3-4.2 \mathrm{~K}^{47}$ (showing potential magnetic phase transitions), and on only wet samples of melanin ${ }^{48}$ with contributions assigned to melanin and water separately. However, as Fig. 1 indicates, one can't separate out water and melanin contributions since water changes the chemistry of 
melanin. Furthermore, if a proper comparison is going to be made with dielectric/AC and DC data, similar temperature ranges need to be covered up to and including room temperature.

It is clear that if the solid-state comproportionation reaction model for conductivity of melanin is to be confirmed via temperature dependent data, certain gaps will need to be filled. Hence, the work presented here addresses the outstanding problems by performing the first broad-band radio-frequency ( $1 \mathrm{~Hz}$ to $1 \mathrm{MHz}$ ) and terahertz $(0.2-3 \mathrm{THz})$ temperaturedependent (5-325 K) measurements of complex AC conductivity and dielectric permittivity of melanin samples with different water content. These studies are supplemented by the first careful measurements of specific heat at temperatures 1.8$300 \mathrm{~K}$. Furthermore, we'll show that utilizing universal scaling the data analysis and understanding of melanin can be significantly enhanced and essentially confirm the comproportionation reaction model for conductivity. Finally, all the work above we will show were done with hydration control in mind, which will demonstrate the importance of controlling not just temperature and frequency, but hydration as well.

\section{Materials and methods}

\section{Sample preparation}

Melanin was synthesized following a standard literature procedure $^{25}$ utilizing as the initial starting material D,L-dopa (Sigma-Aldrich). D,L-dopa was dissolved in deionized water, subsequently adjusted to $\mathrm{pH} 8$ using $\mathrm{NH}_{3}$ (28\%). Air was then bubbled through the solution while being stirred for 3 days. During the 3 days synthesis the $\mathrm{pH}$ would naturally decrease due to the $\mathrm{NH}_{3}$ evaporation, which would necessitate the need to add ammonia periodically to bring the $\mathrm{pH}$ back to 8 . By keeping the $\mathrm{pH}$ at a maximum of 8 and letting it decrease naturally ensured that ring fission of the indolquinone moieties are kept to a minimum and hence the synthesised melanin is a biomimetic material. ${ }^{41}$ The solution was then brought to $\mathrm{pH} 2$ using $\mathrm{HCl}(32 \%)$ to precipitate the pigment. The solution was then filtered and washed multiple times with deionized water and dried. We also note, as per the XPS work below, that our synthetic samples, although a comparable biomimetic analogue to naturally occurring melanin, do have a higher amount of DHI (Fig. 1) moieties relative to DHICA moieties $(\sim 7: 3)$ than what would be expected for naturally occurring melanins which usually exhibit a $1: 1$ ratio of DHI to DHICA. ${ }^{41} \mathrm{~A}$ basic elemental characterization was performed on the sample by use of X-ray Photoelectron Spectroscopy (XPS). Measurements were at the Centre for Microscopy and Microanalysis Xray Analysis Facility (UQ) using a Kratos Axis Ultra XPS Surface Analysis System with a monochromatic Al K $\alpha$ X-ray source. Survey scans were taken at a $120 \mathrm{eV}$ pass energy and analysis performed using CASA XPS software. Atomic ratios for $\mathrm{C}, \mathrm{N}$ and $\mathrm{O}$ were determined and came out at $\mathrm{O} \sim 21.3 \%, \mathrm{C}$ $\sim 70.9 \%$ and $\mathrm{N} \sim 7.9 \%$. These values are commensurate with a polymer based upon $\sim 70 \% \mathrm{DHI}$ monomers. The powders were pressed (30 minutes at 6000 bar) into round pellets with the thickness of 1-2 $\mathrm{mm}$ and diameter $8-10 \mathrm{~mm}$ by means of
LabTools hydraulic press system (Russia). To stabilize the water content the samples were placed then in hygrostats. We controlled the reference values of humidity by means of saturated solutions of $\mathrm{LiCl}, \mathrm{MgCl}_{2}, \mathrm{Na}_{2} \mathrm{Cr}_{2} \mathrm{O}_{7}, \mathrm{NaNO}_{2},\left(\mathrm{NH}_{4}\right)_{2} \mathrm{SO}_{4}$, pure water and dry $\mathrm{P}_{2} \mathrm{O}_{5}$, corresponding to the values of relative humidity at $22{ }^{\circ} \mathrm{C} 11 \%, 33 \%, 55 \%, 65 \%, 98 \%, 100 \%$ and $\sim 0 \%$ respectively. Before and after each measurement procedure (either spectroscopic or specific heat) we weighed the samples and estimated the weight loss or gain against vacuum dry state. Weights measured and correspondence with hygrostats humidities yielded approximate values of water concentration in the samples commensurate with published adsorption isotherms. ${ }^{42}$

\section{Radiofrequency range $\mathrm{AC}$ measurements}

The pellets with the $8-10 \mathrm{~mm}$ diameters and $1.2-1.3 \mathrm{~mm}$ thicknesses were used for the study. Au-electrodes were evaporated on plane-parallel surfaces using BAL-TEC (SCD 050) Sputter Coater. The contacts for applying the electric field were provided by silver wires fixed by a silver (Degussa) paste. Lowfrequency complex dielectric permittivity, losses and conductivity were measured at frequency ( $1 \mathrm{~Hz}$ to $1 \mathrm{MHz}$ ) and temperature (5-295 $\mathrm{K})$ ranges in a standard way using a NOVOCONTROL Alpha-AN High Performance Frequency Analyzer in conjunction with a JANIS ST-100 cryostat controlled by temperature controller Lakeshore 335 . The temperature rate and applied AC electric field was about $0.2 \mathrm{~K} \mathrm{~min}^{-1}$ and $0.83-$ $0.77 \mathrm{~V} \mathrm{~mm}^{-1}$, respectively.

\section{DC-measurements}

DC conductivity was measured in a 4-point van der Pauw geometry with contacts made with silver paint. The voltage was measured with a Keithley 2002 voltmeter (input resistivity above $100 \mathrm{GOhm}$ ) and Keithley 6517A electrometer. The commutator leakage resistance was or the order of TOhm that allows to reliable measure sample resistance of 1-10 GOhm.

\section{Terahertz range $\mathrm{AC}$ measurements}

Terahertz measurements were done using a commercial pulsed terahertz spectrometer TeraView TPS Spectra 3000 (UK) equipped with a home-made helium-flow optical cryostat with Mylar windows.

\section{Specific heat measurements}

The specific heat data were collected by a relaxation technique in a Quantum Design physical property measurement system (PPMS) in the temperature range 1.8-300 K. The samples of a rectangular shape with the mass $\sim 10 \mathrm{mg}$ were used. Dry samples were prepared by means of 72 hours incubation in $\operatorname{Ar}(p$ $\sim 1 \mathrm{~atm})$. Wet samples were made from dry by means of 48 hours incubation in $100 \% \mathrm{RH}$ atmosphere at $25{ }^{\circ} \mathrm{C}$. Water contribution into final mass of the samples was estimated by weighing. Experiments with wet samples started with fast (20 degrees per minute) cooling aimed to minimize water evaporation in helium flow ( $p \sim 5$ torr) inside the cryostat. 


\section{The modelling of the boson peak}

We modelled the low-temperature dependences of the specific heat of our samples via a sum of the Debye contribution and the additional excess vibrational density of states due to a Lorentzian distributed boson peak:

$$
g_{\mathrm{BP}}(\nu) \propto \frac{1}{\left(\nu-\nu_{0}\right)^{2}+\nu_{\delta}^{2}},
$$

where $g_{\mathrm{BP}}(\nu)$ is the frequency dependent density of states for the boson peak, $\nu_{\delta}$ is the frequency width of the boson peak and $\nu_{0}$ is the is the frequency position of the boson peak. The resulting specific heat is then given by a standard expression: ${ }^{43}$

$$
c_{\mathrm{p}} \sim \frac{\mathrm{d}}{\mathrm{d} T}\left[\int_{0}^{\nu_{\mathrm{D}}} g_{\mathrm{D}} \frac{\nu}{\exp \left(\frac{\nu}{T}\right)-1} \mathrm{~d} \nu+\int_{0}^{\nu_{\max }} g_{\mathrm{BP}} \frac{\nu}{\exp \left(\frac{\nu}{T}\right)-1} \mathrm{~d} \nu\right],
$$

where $\nu_{\mathrm{D}}$ is the Debye cutoff frequency is related to the Debye temperature $\Theta_{\mathrm{D}}$ via $h \nu_{\mathrm{D}}=k_{\mathrm{B}} \Theta_{\mathrm{D}}$, (with $k_{\mathrm{B}}$ and $h$ being Boltzmann and Planck's constants, respectively), $\nu_{\max }>\nu_{0}+\nu_{\delta}$. From the peak position we estimate the correlation length $\xi$, that is associated with the spatial scales of elastic constants fluctuations which produce the boson peak on a microscopic scale. Its value is usually defined as the ratio of the transverse sound velocity $v_{\mathrm{s}}$ to the boson peak frequency as $\xi \approx v_{\mathrm{s}} / \nu_{0} \cdot{ }^{44} \mathrm{We}$ calculate the values of $\xi$ with the sound velocity estimated to be $2 \times 10^{5} \mathrm{~cm} \mathrm{~s}^{-1} .^{45-49}$

\section{Experimental results}

\section{Electrical measurement results}

Fig. 2 depicts the radiofrequency-terahertz spectra of the real $\varepsilon^{\prime}(\nu)$ and imaginary $\varepsilon^{\prime \prime}(\nu)$ parts of the permittivity and the real component of the conductivity, $\sigma_{1}(\nu)=\nu \varepsilon^{\prime \prime} / 2$ for two types of melanin sample - one dried in vacuum for 10 hours and another incubated at $48 \% \mathrm{RH}$ at $22{ }^{\circ} \mathrm{C}$, which corresponds to a mass gain of $\sim 13.5 \%$. This level of hydration is sufficient for melanin to be in the conductive regime in which the comproportionation reaction is strongly exhibited. ${ }^{26,30,31} \mathrm{~A}$ strong decrease in the real and imaginary permittivity values are seen at room temperature in the spectra of the wet melanin as one increases the frequency. Such behavior is accompanied by moderate increase of the frequency dependent conductivity $\sigma_{1}(\nu)$. However, when cooled down, the strong dispersion observed for $\varepsilon^{\prime}(\nu)$ and $\varepsilon^{\prime \prime}(\nu)$ weakens significantly and both spectra nearly flatten at around $150 \mathrm{~K}$.

The nearly constant imaginary permittivity, $\varepsilon^{\prime \prime}(\nu) \approx$ const, together with the corresponding conductivity $\sigma_{1}(\nu) \sim \varepsilon^{\prime \prime} \nu \sim \nu^{1}$ behavior indicate emergence of the nearly-constant loss regime frequently observed in biological and disordered systems. ${ }^{50-56}$ In contrast, the dry sample appears to be in a nearly constant loss regime already at room temperatures, with cooling only having a minimal effect. This indicates the clear effect that water has on melanin's electrical properties in promoting a hopping regime for conductivity (see below).

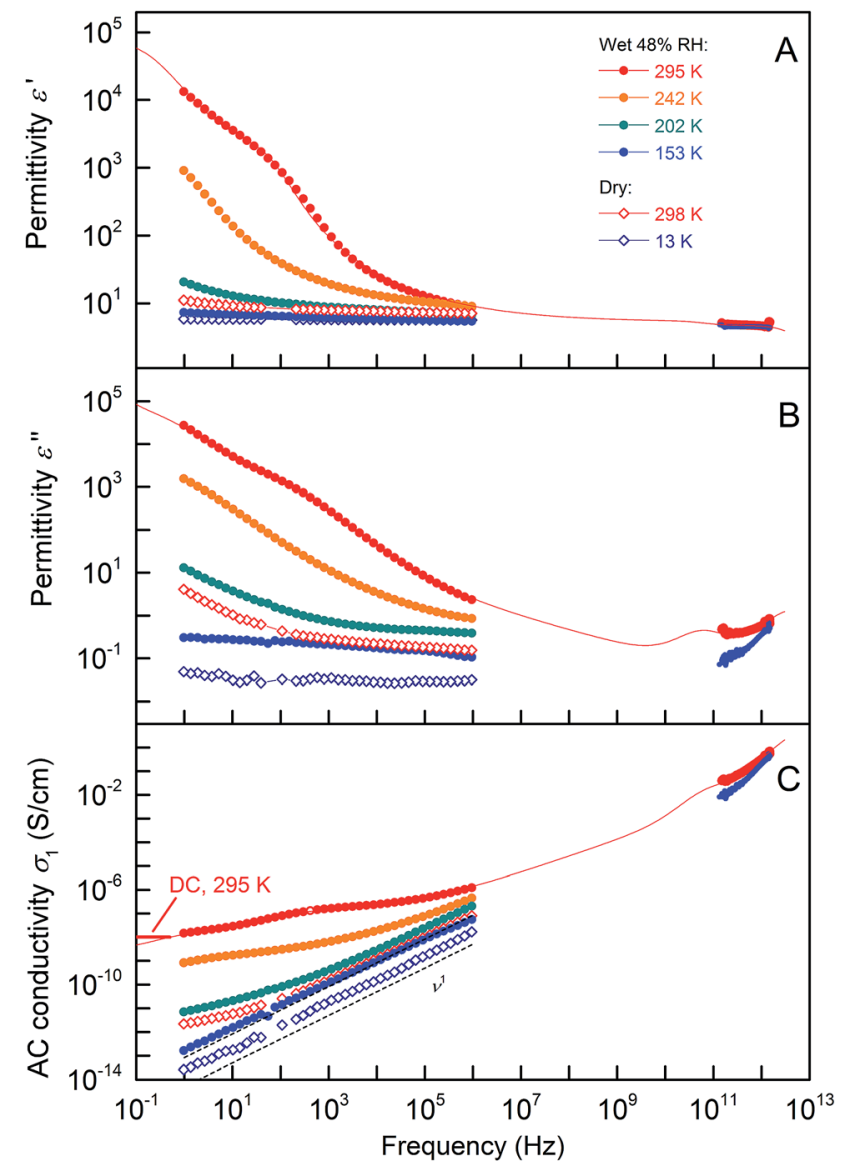

Fig. 2 Radio-frequency and terahertz spectra of (A) real and (B) imaginary dielectric permittivity and (C) AC conductivity at different temperatures as indicated. Circles indicate melanin incubated at $48 \%$ $\mathrm{RH}$ and $22{ }^{\circ} \mathrm{C}$ (containing $\sim 13.5 \%$ wt of water) and rhombuses for melanin dried in vacuum for $\sim 10$ hours. Solid lines correspond to fitting of the room-temperature spectra as described in the text. Dashed line in panel (C) corresponds to the $\sigma_{1} \propto \nu^{\mathrm{s}}, s=1$, dispersion in the nearly-constant loss regime, $\varepsilon^{\prime \prime}(\nu) \sim \sigma_{1} / \nu=$ const. Coincidence of the room-temperature DC conductivity (see (C)) with the AC conductivity signifies absence of any contact effects.

We also note that the temperature dependent features in the permittivities (including similar values and frequency features) show the same qualitative behavior as those for melanin thin films $^{34}$ (see also Fig. $\mathrm{S} 1 \dagger$ ) that are successively hydrated.

The observed temperature evolution of the spectra was processed with the minimal set of appropriate terms. The pronounced dispersion in the high-temperature spectra below 1 $\mathrm{MHz}$ was modeled with the Havriliak-Negami relaxation expression $^{57}$ commonly used to describe frequency dependent response in disordered media:

$$
\varepsilon_{\mathrm{HN}}^{*}(\nu)=\frac{\Delta \varepsilon_{\mathrm{HN}}}{\left[1+\left(i \nu / f_{\mathrm{R}}\right)^{1-\alpha}\right]^{\beta}},
$$

where $\Delta \varepsilon_{\mathrm{HN}}$ is the dielectric contribution of the relaxation, $f_{\mathrm{R}}$ is the relaxation frequency, $\alpha$ and $\beta$ characterize the broadness and asymmetry of the relaxation peak, respectively. The relaxation frequency $f_{\mathrm{R}}$ was estimated to be $\approx 0.2 \mathrm{~Hz}$ and was found 
to be almost temperature independent. To describe the growing over several decades conductivity we had to introduce, additionally to the Havriliak-Negami term, the hopping-like term for conductivity, ${ }^{58}$ that is the only mechanism adequately corresponding to the observed spectral response. The hopping conductivity is given as

$$
\sigma_{1}(\nu)=A \nu^{\mathrm{s}}
$$

And the real permittivity is provided by the Kramers-Kronig relations as

$$
\varepsilon^{\prime}(\nu)=2 A \nu^{s-1} \tan \left(\frac{\pi s}{2}\right)
$$

We also observed a second relaxation process in the room temperature permittivity and conductivity spectra with its $f_{\mathrm{R}}$ estimated around $60 \mathrm{~Hz}$ (bump between 100 and $1000 \mathrm{~Hz}$ in conductivity and imaginary permittivity spectra, see Fig. 2). This second relaxation disappeared during cooling much faster than the $0.2 \mathrm{~Hz}$ feature and could not be observed below $250 \mathrm{~K}$. The characteristic frequencies for both relaxation phenomena exhibited significant thermal stability and have activation energies of $0.43 \mathrm{eV} \pm 10 \%(0.2 \mathrm{~Hz}$ feature $)$ and $0.39 \mathrm{eV} \pm 40 \%$ (60 Hz feature), Fig. 3A. The features have significantly different strengths $\left(\Delta \varepsilon_{\mathrm{HN}}\right)$ of about two orders of magnitude between the $0.2 \mathrm{~Hz}$ and $60 \mathrm{~Hz}$ features at room temperature.

Example of a least-square fit of the radio-frequency and $\mathrm{THz}$ spectra is shown by solid lines in Fig. 2. Same procedure was used to process the spectra of dry sample that are shown in Fig. 2A-C by rhombuses. The terahertz-infrared spectra are described with a Lorentzian (infrared excitation seen as increase in $\varepsilon^{\prime \prime}$ and $\sigma_{1}$ above $10^{12} \mathrm{~Hz}$ ) and Debye (terahertz response of water seen as a peak around $10^{11} \mathrm{~Hz}$ in panel B) models as explained below.

Fig. $3 \mathrm{~A}-\mathrm{C}$ plots the temperature dependences of the above parameters and there is clearly a hydration dependence. The hopping contribution to the radio-frequency response also freezes out while cooling (Fig. 3B). The temperature dependence of the hopping exponent $s$ shows a crossover at 200-240 K from hopping regime $(s=0.7-0.8)$ to the nearly constant loss regime $(s>0.9)$. Usually nearly-constant loss regime in proteins is associated with the dynamics of caged water molecules. ${ }^{55,59}$

In many cases the experimental data on AC/DC conductivity and dielectric permittivity of disordered conductors, obtained at different frequencies and temperatures, can be merged on a single master curve thus signifying the frequency-temperature superposition principle. ${ }^{\mathbf{5 2 , 6 0}}$ Such universal scaling can be revealed by plotting the reduced conductivity or permittivity versus reduced frequency. ${ }^{61}$ Physically, its fulfillment indicates an unchanged mechanism of electrodynamic response (in the present case conductivity) in corresponding frequency and temperature intervals. We plot such a curve in Fig. 4 for the conductivity spectra of the $13.5 \%$ wt melanin according to the relation:

$$
\frac{\sigma_{1}(\nu)}{\sigma_{\mathrm{DC}}}=F\left(\frac{\nu}{\sigma_{\mathrm{DC}} T}\right)
$$

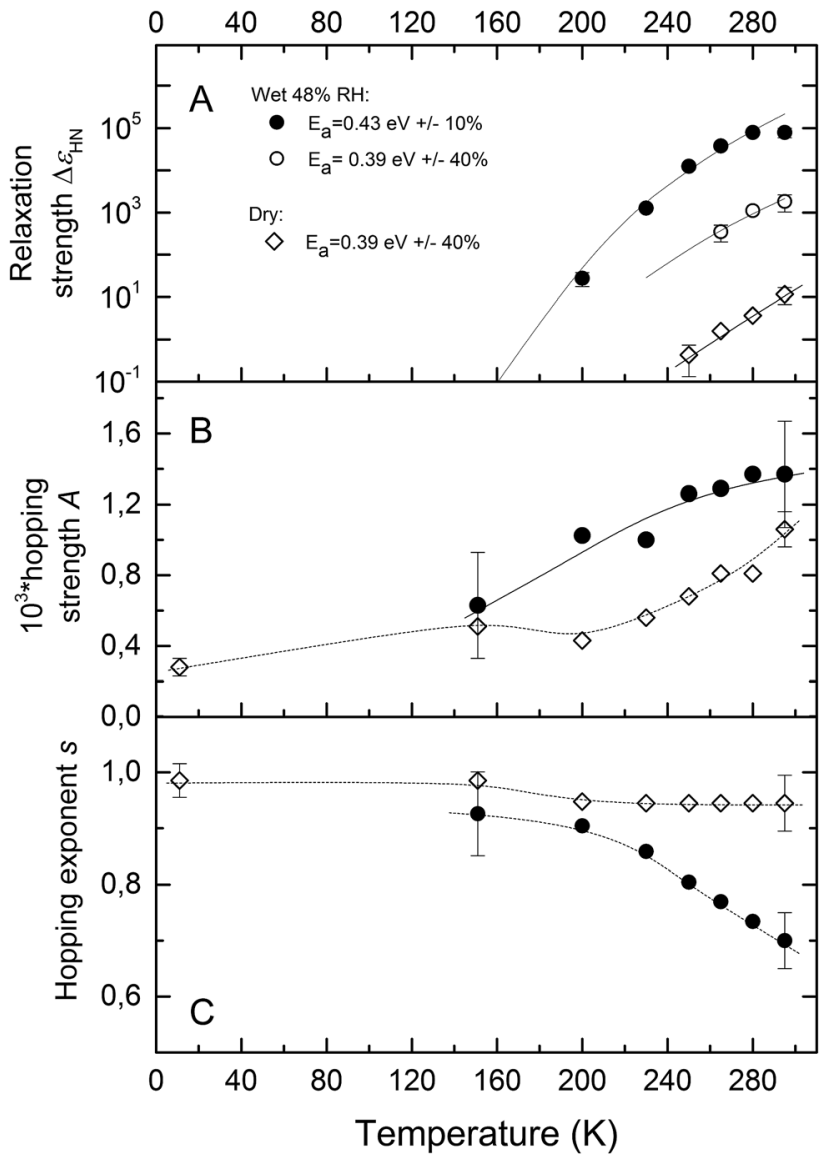

Fig. 3 On panel (A) we present results of temperature variations of relaxation strengths and corresponding activation energies (solid lines) of two processes, observed at frequencies $0.2 \mathrm{~Hz}\left(E_{\mathrm{a}}=0.43 \mathrm{eV} \pm 10 \%\right)$ and $60 \mathrm{~Hz}\left(E_{\mathrm{a}}=0.39 \mathrm{eV} \pm 40 \%\right)$ in dry (empty rhombuses) and wet (empty and filled circles) samples of melanin. On panels (B) and (C) we fitted temperature behavior of hopping strength $\Delta \varepsilon_{\mathrm{HN}}$, eqn (3), and hopping exponent $s$, eqn (4). Dashed lines in panels $B$ and $C$ are guides to the eye.

where $F$ is scaling function. For the room-temperature data, our measured DC conductivity was used. However, since we were unable to obtain $\sigma_{\mathrm{DC}}$ at lower temperatures, $\sigma_{\mathrm{DC}}$ values were chosen to satisfy eqn (6). In essence, we utilized universal scaling to our advantage to infer the conductivity of melanin where we were unable to measure. This technique should prove useful for conductive biomaterials in general. The values of $\sigma_{\mathrm{DC}}$ were then used to obtain a characteristic activation energy $0.65 \mathrm{eV} \pm 15 \%$, which is similar to the activation energy of $0.43 \mathrm{eV} \pm 10 \%$ observed for the peak fitting of the $0.2 \mathrm{~Hz}$ feature. This is not surprising given that such a feature is approaching the DC limit behavior, as one would naturally infer from Fig, 2c (295 K, wet data, low frequency).

As a consistency check on the scaling methodology above, the AC data obtained at $1 \mathrm{~Hz}$ was plotted $v s$. 1/T, and an activation energy of $0.52 \mathrm{eV} \pm 10 \%$ was extracted (Fig. S2, $\dagger$ filled circles). Again, similar to our inferred DC conductivity activation energy, with discrepancies most likely being due to not operating in the DC limit. For completeness, we include the 


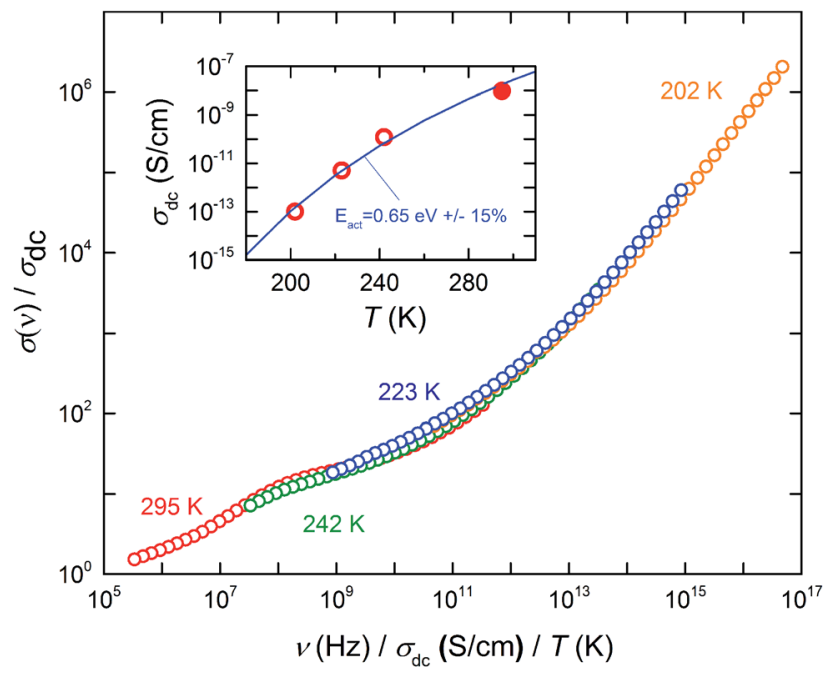

Fig. 4 Scaling curve in accordance with eqn (6) for conductivity data of the melanin sample containing $\sim 13.5 \%$ wt of water. Different colors indicate different temperatures. The inset demonstrates that the DC conductivity $\sigma_{\mathrm{DC}}$ shows activating behavior with an activation energy of $0.65 \mathrm{eV} \pm 15 \%$. Filled dot corresponds to measured value; open dots correspond to the values obtained from scaling analysis as described in the text. Experimental uncertainties are of the data points size.

$1 \mathrm{~Hz}$ data obtained for the dry sample with the associated activation energy of $0.43 \mathrm{eV} \pm 10 \%$, which is similar to the activation energies obtained for the $60 \mathrm{~Hz}$ feature in the vacuum level data (Fig. $\mathrm{S} 2 \dagger$ ).

It should also be noted that we do not observe any sign of relaxation processes described and interpreted by Jastrzebska et $a .^{39}$ as a sign of two types of hopping phenomena. However, we did not heat our samples to as high temperatures as reported by them. ${ }^{39}$

To model the terahertz spectra (included in Fig. 2) we used a Lorentzian expression that describes the resonance located above our highest frequency, and the Debye expression ${ }^{56}$ to reproduce the response of water molecules (free or bound) clearly seen at $T=325 \mathrm{~K}$ in the spectra of Fig. 5 (note the changes in the value is non zero). The Debye expression used is given by:

$$
\varepsilon_{\mathrm{D}}^{*}(\nu)=\frac{\Delta \varepsilon_{\mathrm{D}}}{1+\mathrm{i}\left(\nu / \gamma_{\mathrm{D}}\right)},
$$

where $\Delta \varepsilon_{\mathrm{D}}$ is relaxation strength and $\gamma_{\mathrm{D}}$ is the relaxation rate. Strong sensitivity to water of the THz spectra is demonstrated in Fig. 5, where the permittivity spectra of a wet melanin sample are shown $\left(\sim 14.7 \mathrm{wt} \%\right.$ at $\left.22^{\circ} \mathrm{C}\right)$. At high enough temperatures (250 K and above) a strong increase towards low frequencies of $\varepsilon^{\prime}(\nu)$ and flattening of $\varepsilon^{\prime \prime}(\nu)$ spectra can be seen. Both features are strongly indicative of a relaxational response of water molecules with a characteristic relaxation frequency located below our working $\mathrm{THz}$ interval. Note that in the absence of mentioned relaxational feature the real and imaginary permittivities would show $\varepsilon^{\prime} \rightarrow$ const and $\varepsilon^{\prime \prime} \rightarrow 0$ at $\nu \rightarrow 0$. This result acts as an independent confirmation that significant amounts of liquid like water is present in the wet melanin sample at high

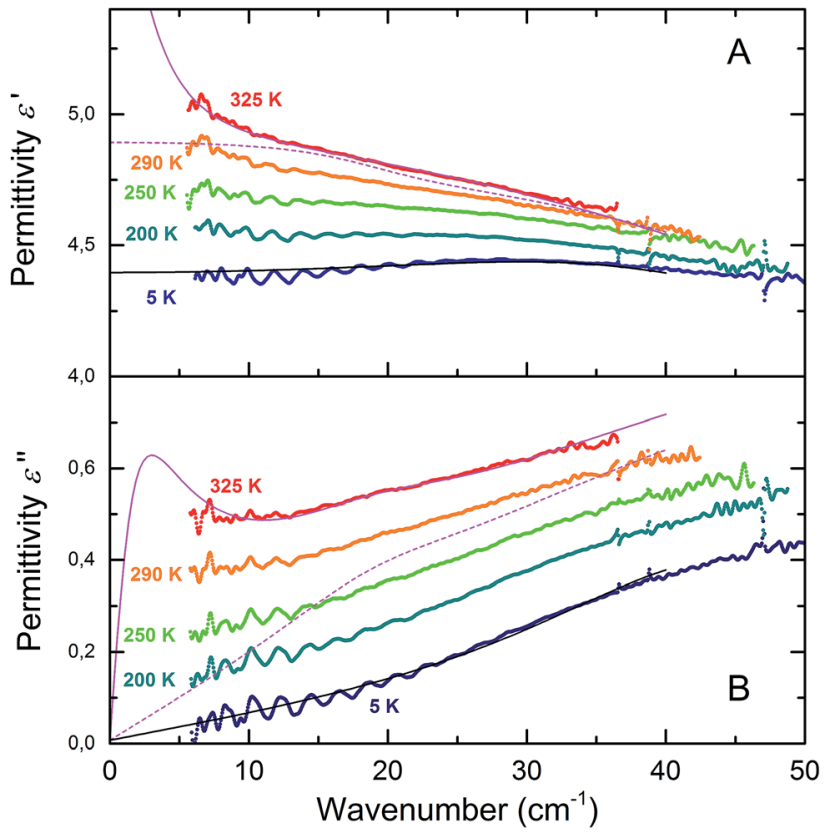

Fig. 5 The temperature dependent terahertz spectra of real (A) and imaginary (B) parts of the permittivity of wet melanin ( 14.7\% wt). Solid magenta lines fits the $T=325 \mathrm{~K}$ spectra with the Lorentzian term responsible for the infrared absorption and Debye term describing the response of water $\left(\Delta \varepsilon \approx 3, \gamma_{D} \approx 1 \mathrm{~cm}^{-1}\right)$ as discussed in the text. Dashed lines is the fit without the Debye term. Black solid lines show fits of the spectra obtained for $T=5 \mathrm{~K}$.

temperatures. We note that the hopping regime elucidated above (Fig. 3C) dominates at $250 \mathrm{~K}$ and above, as well, hence suggesting that liquid like water is a necessary component for conductivity.

\section{Heat capacity measurements results}

The results of our specific heat measurements of dry and wet (15-20 wt\% of water) melanin are presented in Fig. 6 . As can be seen, at temperatures below $3.5 \mathrm{~K}$ and above $\approx 20 \mathrm{~K}$ the wet sample has a larger specific heat compared to the dry sample. This effect is more clearly demonstrated in the $c_{\mathrm{P}}(T) / T^{3}$ vs. $T$ presentation (Fig. 6B), where both dry and wet melanin types reveal a feature typical for classic amorphous systems - a boson peak. The boson peak is observed in disordered materials in the form of an excess contribution to the vibrational density of states (VDOS) $g(\nu)$, relative to the Debye component $g_{\mathrm{D}}(\nu) \sim \nu^{2}$. This additional contribution can be observed by inelastic neutron or Raman scattering ${ }^{62-67}$ and as a peak in the specific heat plotted as $c_{\mathrm{P}}(T) / T^{3} v s . T^{56,68}$

Furthermore, there is a remarkable observation which comes from the inset on Fig. 6A. Within the framework of our techniques the specific heat of dry melanin goes to zero as we approach to $0 \mathrm{~K}$, unlike specific heat of wet melanin. This suggests that dry melanin has a more regular, less disordered structure. Furthermore, we also observe that at temperatures above $100 \mathrm{~K}$, again unlike in wet sample, we can clearly see a gradual decrease of the dry melanin heat capacity (Fig. 6), 

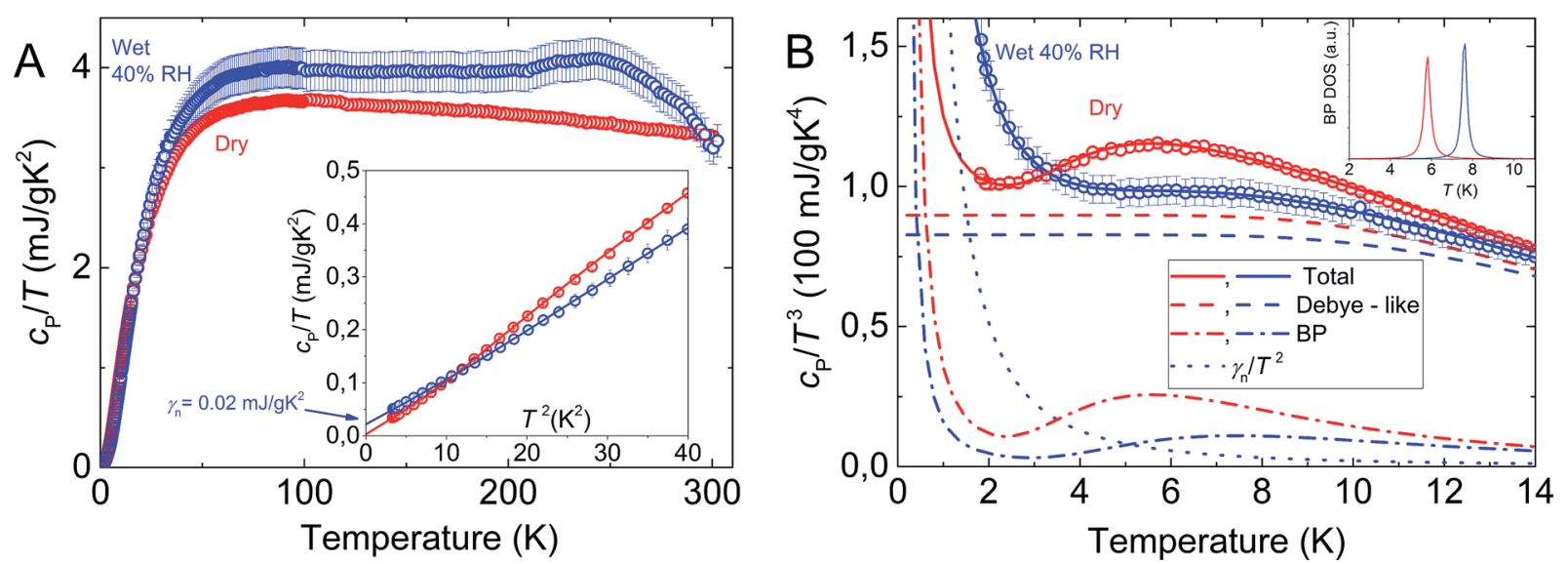

Fig. 6 Temperature dependences of specific heat of dry and wet (15-20 wt\% of water) melanin. (A) - $C_{P} / T$ of dry melanin (red) and wet melanin (blue). The inset shows $C_{P} / T$ versus $T^{2}$ at low temperatures, $C_{P} / T$ of wet melanin does not go to zero at $T=0 \mathrm{~K}$; symbols - experimental data and solid lines fits by the model eqn (2); $\gamma$ is addition to Debye contribution. Experimental uncertainty is of the data points size. (B) $-c_{p} / T^{3}-T$ representation of specific heat data. Symbols - experimental data with solid lines showing the model of eqn (2). Dashed lines show Debye contribution. Dashed-dotted lines show boson peak (BP) contribution and dotted lines show linear contribution. The inset shows the BP DOS having Lorentzian distribution. BP feature (peak at $\sim 6 \mathrm{~K}$ ) is clearly seen in dry melanin and its shift to a higher temperature in wet melanin.

which speaks in favor of structural reforming within the system. We will discuss this in more detail in the discussion below.

We also note that the heat capacity does not go to 0 as we approach $0 \mathrm{~K}$ in the case of wet melanin thus pushing us to add linear component to standard boson peak and Debye-like terms which are enough for description of dry sample behavior.

\section{Discussion}

\section{The $0.2 \mathrm{~Hz}$ feature}

The current study revealed several phenomenological peculiarities that have not been observed earlier. For example, two low frequency relaxations with characteristic frequencies at $\sim 0.2 \mathrm{~Hz}$ and $\sim 60 \mathrm{~Hz}$ have not been reported before. To explain the origin of these features one needs to recall both melanin's solid-state chemistry and physical structure. We will focus on the $0.2 \mathrm{~Hz}$ peak first and note three key features of the $0.2 \mathrm{~Hz}$ response, which are: (a) has a relaxation frequency of $0.2 \mathrm{~Hz}$, hence the physical size/mass of the electric dipole involved must be large to account for the slow response; (b) only seen in hydrated melanin; (c) has an activation energy of $0.43 \mathrm{eV} \pm 10 \%$. The most parsimonious explanation accounting for these observations is to assign the $0.2 \mathrm{~Hz}$ relaxation to the semiquinone anions within melanin. Dealing with point (a) first, the reality is that the semiquinone moieties with melanin will be part of an oligomer sheet of 4-6 monomers, ${ }^{20,69-73}$ which in turn will stack with other such sheets via the $\pi-\pi$ interaction ${ }^{69,74}$ to form a colloidal particle. Hence, the charged electric dipole of the semiquinone, in order to align itself with the electric field, will have to rotate a relatively larger colloidal particle, leading to a slow relaxation frequency.

Given that the comproportionation reaction, and hence the semiquinone, is mostly exhibited in hydrated melanins ${ }^{26}$ due to the requirement of two water molecules to generate them, it addressed point (b) and explains why this $0.2 \mathrm{~Hz}$ feature is not present in the dry sample.
The above leads us directly to point (c), in order to explain the origin of the activation energy, it is necessary to recall the equilibrium constant for the overall comproportionation reaction:

$$
K_{\text {eq, comp }}=\frac{a_{\mathrm{H}_{3} \mathrm{O}^{+}}{ }^{2} a_{\mathrm{SQ}^{-}}}{a_{\mathrm{H}_{2} \mathrm{Q}} a_{\mathrm{Q}} a_{\mathrm{H}_{2} \mathrm{O}^{2}}}=\mathrm{e}^{-\frac{\mathrm{r}_{\mathrm{r}} G_{\mathrm{comp}}}{R T}}
$$

where $a_{\mathrm{x}}^{y}$ is the activity for a particular chemical species, $\mathrm{H}_{2} \mathrm{Q}$ refers to hydroquinone, $\mathrm{Q}$ to quinone, $\mathrm{SQ}^{-}$refers to the semiquinone, $\mathrm{H}_{2} \mathrm{O}$ is for water, $\mathrm{H}_{3} \mathrm{O}^{+}$refers to hydronium, $R$ is the universal gas constant and $\Delta_{\mathrm{r}} G_{\text {comp }}$ is the Gibbs free energy of reaction for the comproportionation equilibrium. $\Delta_{\mathrm{r}} G_{\text {comp }}$ magnitude can be estimated (see $\mathrm{ESI} \dagger$ ) from the known underlying monomer chemistry of dihydroxyindole, a building block of melanin, and comes in around $1.08 \mathrm{eV}$. If one makes the approximation that:

$$
\left[\mathrm{SQ}^{-}\right] \approx a_{\mathrm{SQ}^{-}} \propto \sqrt{K_{\text {eq, comp }}}=\mathrm{e}^{-\frac{\Delta_{\mathrm{r}} G_{\text {comp }}}{2 R T}},
$$

which implies that the concentration variation of the semiquinones will have an activation energy of approximately $\Delta_{\mathrm{r}^{-}}$ $G_{\text {comp }} / 2$, or $0.54 \mathrm{eV}$. Since $\Delta \varepsilon$, the relaxation strength, is related to the number of dipoles, i.e. semiquinone concentration, it can be seen that the activation energy determined from Fig. 3a for the $0.2 \mathrm{~Hz}$ relaxation shows remarkable similarity to what one would estimate from the comproportionation reaction equilibrium chemistry.

That the comproportionation reaction is the origin of the $0.2 \mathrm{~Hz}$ feature is bolstered by the fact that the temperature dependent trend of the dielectric parameters have the same behavior as the hydration dependent trend reported elsewhere ${ }^{34}$ (Fig. S1†). This argues for the same underlying mechanism i.e. the comproportionation reaction.

Indeed, if the above interpretation is adopted, unusual consistency can be obtained when inspecting the scaled DC 
conductivity data (Fig. 4). Since melanin is a proton conductor ${ }^{34}$ who's conductivity is due to charge density changes of protons regulated by the comproportionation reaction, one can write

$$
\sigma_{\mathrm{DC}} \propto n_{\text {protons }} \propto\left[\mathrm{H}_{3} \mathrm{O}^{+}\right]=\left[\mathrm{SQ}^{-}\right] .
$$

Hence, one would anticipate that the conductivity would show the same order of magnitude in its activation energy as for the dielectric relaxation at $0.2 \mathrm{~Hz}$. Given that $0.65 \mathrm{eV} \pm 15 \%$ is recorded for the DC conductivity, it is again confirmation of the consistency of the above interpretation.

In short, the data, both conductivity and dielectric spectra, confirms the presence of the comproportionation reaction, but for the first time is done so utilizing the temperature variable.

\section{The $60 \mathrm{~Hz}$ feature}

Our above explanation inclines us to believe that energies around the $0.5 \mathrm{eV}$ value may be signature values for chemical equilibria. Hence, with this mind set we approached the $60 \mathrm{~Hz}$ relaxation feature by noting the following key features: it is higher in frequency than the $0.2 \mathrm{~Hz}$ feature, is present in both the dry and wet melanin forms, it is smaller in magnitude to the $0.2 \mathrm{~Hz}$ feature, and it has a lower energy of activation of $0.39 \mathrm{eV}$ $\pm 40 \%$ than the $0.2 \mathrm{~Hz}$ feature. Considering the higher frequency of the relaxation, it implies that the electric dipole entity is spatially/mass wise smaller in magnitude. Furthermore, the smaller magnitude of the relaxation strength implies far fewer entities than the semiquinone. The best explanation for these observations is to assign the $60 \mathrm{~Hz}$ feature to the carboxylic acid groups present in melanin. First, they are smaller entities and hence easier to polarize, leading to a higher relaxation frequency. Furthermore, there is only a few $\mathrm{COOH}$ moieties present in our samples, which explains the lower dielectric strength. One can also write:

$$
K_{\mathrm{a}}=\frac{a_{\mathrm{H}_{3} \mathrm{O}^{+}} a_{\mathrm{COO}^{-}}}{a_{\mathrm{COOH}} a_{\mathrm{H}_{2} \mathrm{O}}}=\mathrm{e}^{-\frac{\Delta_{\mathrm{r}} G_{\mathrm{a}}}{R T}},
$$

and

$$
\left[\mathrm{H}_{3} \mathrm{O}^{+}\right] \approx a_{\mathrm{H}_{3} \mathrm{O}^{+}} \propto K_{\mathrm{a}}
$$

where $\mathrm{COOH}$ indicates the protonated carboxylic group, $\mathrm{COO}^{-}$ indicates the deprotonated carboxylic acid group and $\Delta_{\mathrm{r}} G_{\mathrm{a}}$ is the Gibbs free energy of reaction for the acid dissociation. With a $\mathrm{p} K_{\mathrm{a}}$ of $4.25(6)$ for DHICA, ${ }^{75}$ this leads to an activation energy of $0.25 \pm 0.1 \mathrm{eV}$. This falls within uncertainty of the estimated 0.39 $\pm 40 \% \mathrm{eV}$.

If the above view is adopted, it can also explain why this $60 \mathrm{~Hz}$ feature is present in both dry and wet melanin. The first is that dry melanin usually still have trace amounts of water ${ }^{20,42}$ and since only one water molecule is required for ionization, and there is a lower activation energy involved, observing it should not be a surprise. Naturally, water is plentiful in the wet melanin sample, hence why one observed it there.

This may also explains the activation energy observed in the $1 \mathrm{~Hz}$ conductivity data (Fig. S2 $\dagger$ ) for the dry melanin. The lower

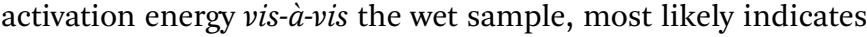
the activation energy of the carboxylic acid dissociation.

An intriguing conclusion can be drawn. The carboxylic acids present do not contribute much to the proton conductivity of melanin. They are too few in number and the conductivity is clearly dominated by a higher activation energy process (i.e. the comproportionation reaction). This has been assumed to be the case in past discussion in various works, but the data presented here confirms it experimentally.

\section{Conductivity and terahertz results}

With the $0.2 \mathrm{~Hz}, 60 \mathrm{~Hz}$ and conductivity data explained, we move to the nature of the conductivity mechanism. For wet melanin, the hopping exponent $s$ (see eqn (4)) increases from $\sim 0.7$ to $\sim 0.9$ with decreasing temperature. These values are indicative of a hopping mechanism. In addition, as noticed in the correlation with the terahertz data in Fig. 5, liquid like water appears to be necessary for this hopping mechanism, which though slowly becomes frozen out as one passes $160 \mathrm{~K}$. The conductivity mechanism, for the wet melanin sample, then enters the range of values similar to the dry melanin, implying a convergence in mechanistic behavior.

Considering that melanin is a proton conductor, it is not unreasonable to assume a Grötthuss mechanism ${ }^{76}$ as the hopping mechanism observed in the wet sample. As the sample is cooled the amount of liquid, hence easily rotatable molecules, becomes less. This leads to a freezing out of the hopping mechanism at low temperature. The low temperature nearly constant loss regime can then tentatively be assumed to indicate a mass of center diffusion mechanism or a much slower hopping mechanism, which will also be present in the dry sample with characteristic activation energy of $0.026 \mathrm{eV} \pm 20 \%$.

\section{Heat capacity results}

We discuss the boson peak behavior first (Fig. 6B). Peculiarities of boson peak behavior in proteins and other organic glassformers have already been intensively studied by the group of Sokolov. ${ }^{77,78}$ They demonstrated that the boson peak shape should not be directly associated with specific chemical composition and chemical bond type of particular glass-former, but is related to general features common to glasses and proteins. Currently, they ascribe the universality of boson peak phenomena to nanoheterogeneity models. ${ }^{78}$ Namely, the density of states spectrum is determined by the spatial distribution of fluctuations of elastic constants of the amorphous structure. In turn, these can be considered as universalities observed in a wide class of materials. The effect of boson peak shifting to higher temperatures with increase of water content (inset in Fig. 6B) is also well known for proteins. Precise analysis of this effect for the case of lysozyme can be found in (ref. 77): increase of hydration results in shifting of the boson peak to higher temperatures, owing to an increase of contribution coming from the fast (picoseconds) relaxation processes associated with the "rattling" of amino acids within cages formed by neighboring residues and solvent molecules. In the case of 
melanin, carboxylic acid moieties rotation and vibration are a plausible source, especially if they are hydrated.

Turning to Fig. 6A and its peculiar features. We first note the low temperature regime (inset), where the dry melanin limits to no heat capacity at $0 \mathrm{~K}$, while the wet melanin sample emphatically have a non-zero intercept. Traditionally, for well ordered, non-conductive systems, the heat capacity limits to zero as one approaches $0 \mathrm{~K}$ since all the phonon modes are frozen out. A non-zero intercept is then indicative of free carriers. Naturally, given that melanin is an amorphous proton conducting material, this interpretation would normally give way to an alternative viewpoint: that the non-zero intercept is a common observation for disordered systems, and is related to lattice vibrations with trapped cavities included ${ }^{64}$ However, this latter view also faces difficulties since the dry melanin sample should then also demonstrate a non-zero intercept for the heat capacity.

In short, the indirect signatures of the possible presence of free carriers in wet melanin both at high (scaling on Fig. 4) and at low (linear component of specific heat on Fig. 6) temperatures are obviously not enough to regard melanin as a system with free electrons and/or protons in a wide temperature range. Unfortunately, we were not able to measure $\sigma_{\mathrm{DC}}$ and estimate $\sigma_{1}$ in radiofrequency range of the wet melanin (with 15-20\% wt of water) at the lowest temperatures. The latter one can, possibly, give us dispersionless behavior as a clear signature of free Drude-like carriers in the system. Thus, these points indicate the need for further study to further elucidate the low temperature heat capacity behavior.

Finally, we turn to the high temperature heat capacity data for melanin. For the wet melanin above $100 \mathrm{~K}$, the behavior is as expected. From $100 \mathrm{~K}$ to $\sim 200 \mathrm{~K}$ the heat capacity/temperature data is constant as would be expected for the Dulong-Petit law. The changes in the heat capacity afterwards is due to water melting and then evaporation. In contrast, the dry melanin data is irregular. In the $100 \mathrm{~K}$ and above data, there is decrease in the data line, violating the Dulong-Petit law. This may be due to a structural change within the dry melanin. It suggests that dry melanin has, as it is being heated, becoming more disordered, hence implying a more "ordered" state. Furthermore, this "ordered" state should be absent in the hydrated polymer. As such, we tentatively advance the idea that the effects seen is due to the formation of quinhydrone pairs at lower temperatures. The quinhydrone hypothesis for melanin has been discussed in the past but has fallen out of favor in recent decades (see discussion in the ESI $†$ ). On the one hand, the formation of quinhydrone dimers (as seen in Fig. 1, pathways I, II and III) should lead to greater regularity of the system at low temperature. But on the other hand it should also significantly destroy the general network of H-bonds, leading to higher energy barriers for proton conduction within material.

\section{Implications for melanin based devices}

The biomimetic synthetic analogue of melanin used in this study has potential application in bioelectronic devices as was indicated in the introduction. Since it is made synthetically, and hence there is more control, applying it to devices is a natural way to go. The present study touches on the fundamental questions of charge transport and mechanism of melanin and hence will inform what kind of devices are feasible in principle using our material. Our results clearly align with the view that the redox chemistry of melanin, via the comproportionation reaction, is responsible for the conductivity of melanin. Hence, considering melanin as a semiconductor is out of the question and thus attempting to create devices such as field effect transistors from melanin are most likely not feasible, since such devices require a semiconductor material.

In contrast, melanin based devices utilising its ionic behaviour have a future, as recent work on batteries, ${ }^{16,17}$ capacitors ${ }^{14}$ and organic electrochemical transistors ${ }^{12}$ prove. Furthermore, as our work would indicate, the carboxylic acid groups do not contribute much to the charge transport, hence focus in creating different kinds of biomimetic melanins in order to control the relative DHI/DHICA content (i.e. controlling the carboxylic acid content) may not offer great advantages as far as proton charge density is concerned. However, this does not discount the importance of the ratio when it comes to secondary structural formation, ${ }^{79}$ which may still strongly influence charge transport in other aspects.

\section{Conclusions}

In conclusion, we have presented the first systematic temperature controlled DC, AC conductivity/permittivity/dielectric spectroscopy and heat capacity measurements on the synthetic melanin while controlling its hydration state appropriately.

The control of these variables was key in demonstrating the potential connection between the hydration dependent behavior for melanin and the temperature dependent behavior. Consistency is achieved with the current proposed indolequinone molecular model where the redox comproportionation reaction is believed to play a dominant role. The data shown here is the first demonstration of the self-consistency of this interpretative model in solid-state melanin using the temperature axis. Furthermore, we were able to pick up the signature of a potential mechanistic competitor in the form of the deprotonation of the carboxylic acid groups in melanin and show that it plays only a minor role. In addition, it was shown that the hopping mechanism in melanin most likely requires liquid water to operate.

\section{Data availability}

The data that support the findings of this study are available from the authors on reasonable request.

\section{Conflicts of interest}

There are no conflicts of interest to declare. 


\section{Acknowledgements}

We acknowledge fruitful discussions with Paul Meredith, Sergei Tretiak, Andriy Zhugayevych. We thank Margarita Sheliakina for help in melanin synthesis and Barry Wood in helping obtain the XPS data. The research was supported by the project N3.9896.2017/BY and Ministry of Education and Science of the Russian Federation (Program 5 top100). ABM is a Sêr Cymru II fellow and results incorporated in this work have received funding from the European Union's Horizon 2020 research and innovation program under the Marie Skłodowska-Curie grant agreement No 663830.

\section{References}

1 A. Noy, Adv. Mater., 2011, 23, 807-820.

2 J. Rivnay, S. Inal, A. Salleo, R. M. Owens, M. Berggren and G. G. Malliaras, Nat. Rev. Mater., 2018, 3, 17086.

3 Y. Tan, R. Y. Adhikari, N. S. Malvankar, S. Pi, J. E. Ward, T. L. Woodard, K. P. Nevin, Q. Xia, M. T. Tuominen and D. R. Lovley, Small, 2016, 12, 4481-4485.

4 N. S. Malvankar and D. R. Lovley, ChemSusChem, 2012, 5, 1039-1046.

5 N. L. Ing, T. D. Nusca and A. I. Hochbaum, Phys. Chem. Chem. Phys., 2017, 19, 21791-21799.

6 K. M. Leung, G. Wanger, M. Y. El-Naggar, Y. Gorby, G. Southam, W. M. Lau and J. Yang, Nano Lett., 2013, 13, 2407-2411.

7 D. D. Ordinario, L. Phan, W. G. Walkup IV, J. M. Jocson, E. Karshalev, N. Hüsken and A. A. Gorodetsky, Nat. Chem., 2014, 6, 596-602.

8 N. Amdursky, X. Wang, P. Meredith, D. D. C. Bradley and M. M. Stevens, Adv. Mater., 2016, 28, 2692-2698.

9 C. Zhong, Y. Deng, A. F. Roudsari, A. Kapetanovic, M. P. Anantram and M. Rolandi, Nat. Commun., 2011, 2, 476.

10 P. Meredith, C. J. Bettinger, M. Irimia-Vladu, A. B. Mostert and P. E. Schwenn, Rep. Prog. Phys., 2013, 76, 034501.

11 J. M. Gallas, Optical lens system incorporating melanin as an absorbing pigment for protection against electromagnetic radiation, US Pat., US 5036115 A, 1991.

12 M. Sheliakina, A. B. Mostert and P. Meredith, Mater. Horiz., 2018, 5, 256-263.

13 M. Ambrico, P. F. Ambrico, A. Cardone, T. Ligonzo, S. R. Cicco, R. Di Mundo, V. Augelli and G. M. Farinola, Adv. Mater., 2011, 23, 3332-3336.

14 P. Kumar, E. Di Mauro, S. Zhang, A. Pezzella, F. Soavi, C. Santato and F. Cicoira, J. Mater. Chem. C, 2016, 4, 95169525.

15 M. Piacenti da Silva, J. C. Fernandes, N. B. de Figueiredo, M. Congiu, M. Mulato and C. F. de Oliveira Graeff, AIP Adv., 2014, 4, 037120.

16 Y. J. Kim, W. Wu, S.-E. Chun, J. F. Whitacre and C. J. Bettinger, Proc. Natl. Acad. Sci. U. S. A., 2013, 110, 20912-20917.

17 C. J. Bettinger and J. Whitacre, A water-activated, ingestible battery, US Pat., US20150118526A1, 2015.

18 G. Prota, Melanins and melanogenesis, Academic Press, 1992.
19 J. Y. Lin and D. E. Fisher, Nature, 2007, 445, 843-850.

20 P. Meredith and T. Sarna, Pigm. Cell Res., 2006, 19, 572-594.

21 T. Sarna, B. Pilas, E. J. Land and T. G. Truscott, Biochim. Biophys. Acta, Gen. Subj., 1986, 883, 162-167.

22 L. Panzella, G. Gentile, G. D'Errico, N. F. Della Vecchia, M. E. Errico, A. Napolitano, C. Carfagna and M. d'Ischia, Angew. Chem., Int. Ed., 2013, 52, 12684-12687.

23 L. Hong and J. D. Simon, J. Phys. Chem. B, 2007, 111, 79387947.

24 S. B. Rienecker, A. B. Mostert, G. Schenk, G. R. Hanson and P. Meredith, J. Phys. Chem. B, 2015, 119, 14994-15000.

25 C. C. Felix, J. S. Hyde, T. Sarna and R. C. Sealy, J. Am. Chem. Soc., 1978, 100, 3922-3926.

26 A. B. Mostert, S. B. Rienecker, C. Noble, G. R. Hanson and P. Meridith, Sci. Adv., 2018, 4, eaaq1293.

27 S.-S. Chio, J. S. Hyde and R. C. Sealy, Arch. Biochem. Biophys., 1980, 199, 133-139.

28 S.-S. Chio, J. S. Hyde and R. C. Sealy, Arch. Biochem. Biophys., 1982, 215, 100-106.

29 A. B. Mostert, G. R. Hanson, T. Sarna, I. R. Gentle, B. J. Powell and P. Meredith, J. Phys. Chem. B, 2013, 117, 4965-4972.

30 A. B. Mostert, B. J. Powell, F. L. Pratt, G. R. Hanson, T. Sarna, I. R. Gentle and P. Meredith, Proc. Natl. Acad. Sci. U. S. A., 2012, 109, 8943-8947.

31 A. Bernardus Mostert, B. J. Powell, I. R. Gentle and P. Meredith, Appl. Phys. Lett., 2012, 100, 093701.

32 M. M. Jastrzebska, H. Isotalo, J. Paloheimo and H. Stubb, J. Biomater. Sci., Polym. Ed., 1995, 7, 577-586.

33 J. Wünsche, Y. Deng, P. Kumar, E. Di Mauro, E. Josberger, J. Sayago, A. Pezzella, F. Soavi, F. Cicoira, M. Rolandi and C. Santato, Chem. Mater., 2015, 27, 436-442.

34 M. Sheliakina, A. B. Mostert and P. Meredith, Adv. Funct. Mater., 2018, 1805514.

35 J. E. McGinness, P. Corry and P. Proctor, Science, 1974, 183, 853-855.

36 C. H. Culp, D. E. Eckels and P. H. Sidles, J. Appl. Phys., 1975, 46, 3658-3660.

37 J. E. McGinness, Science, 1972, 177, 896-897.

38 A. Grebenko, V. Dremov, P. Barzilovich, A. Bubis, K. Sidoruk, T. Voeikova, Z. Gagkaeva, T. Chernov, E. Korostylev, B. Gorshunov and K. Motovilov, PLoS One, 2018, 13, e0191289.

39 M. Jastrzebska, A. Kocot, J. K. Vij, J. Zalewska-Rejdak and T. Witecki, J. Mol. Struct., 2002, 606, 205-210.

40 M. M. Jastrzebska, S. Jussila and H. Isotalo, J. Mater. Sci., 1998, 33, 4023-4028.

41 M. d'Ischia, K. Wakamatsu, A. Napolitano, S. Briganti, J.-C. Garcia-Borron, D. Kovacs, P. Meredith, A. Pezzella, M. Picardo, T. Sarna, J. D. Simon and S. Ito, Pigm. Cell Melanoma Res., 2013, 26, 616-633.

42 A. B. Mostert, K. J. P. Davy, J. L. Ruggles, B. J. Powell, I. R. Gentle and P. Meredith, Langmuir, 2010, 26, 412-416.

43 C. Kittel, Introduction to Solid State Physics, John Wiley, New York, 6th edn, 1986.

44 V. K. Malinovsky, V. N. Novikov, A. P. Sokolov and V. A. Bagryansky, Chem. Phys. Lett., 1988, 143, 111-114. 
45 S. Speziale, F. Jiang, C. L. Caylor, S. Kriminski, C. Zha, R. E. Thorne and T. S. Duffy, Biophys. J., 2003, 85, 3202-3213. 46 J. Griesbauer, A. Wixforth and M. F. Schneider, Biophys. J., 2009, 97, 2710-2716.

47 U. Mizutani, T. B. Massalski, J. E. McGinnes and P. M. Corry, Nature, 1976, 259, 505-507.

48 I.-S. Yang and A. C. Anderson, Phys. Rev. B: Condens. Matter Mater. Phys., 1986, 34(4), 2942-2944.

49 K. Yoshida, A. Q. R. Baron, H. Uchiyama, S. Tsutsui and T. Yamaguchi, J. Chem. Phys., 2016, 144, 134505.

50 B. Roling, C. Martiny and S. Murugavel, Phys. Rev. Lett., 2001, 87, 085901.

51 D. L. Sidebottom and C. M. Murray-Krezan, Phys. Rev. Lett., 2002, 89, 195901.

52 J. C. Dyre, P. Maass, B. Roling and D. L. Sidebottom, Rep. Prog. Phys., 2009, 72, 046501.

53 K. Kämpf, B. Kremmling and M. Vogel, Phys. Rev. E, 2014, 89, 032710.

54 S. Capaccioli, K. L. Ngai, S. Ancherbak and A. Paciaroni, J. Phys. Chem. B, 2012, 116, 1745-1757.

55 K. L. Ngai, S. Capaccioli and A. Paciaroni, Biochim. Biophys. Acta, 2016, 1861, 3553-3563.

56 K. A. Motovilov, M. Savinov, E. S. Zhukova, A. A. Pronin, Z. V. Gagkaeva, V. Grinenko, K. V. Sidoruk, T. A. Voeikova, P. Y. Barzilovich, A. K. Grebenko, S. V. Lisovskii, V. I. Torgashev, P. Bednyakov, J. Pokorný, M. Dressel and B. P. Gorshunov, Sci. Rep., 2017, 7, 15731.

57 S. Havriliak and S. Negami, Polymer, 1967, 8, 161-210.

58 A. K. Jonscher, Dielectric Relaxation in Solids, Chelsea Dielectrics Press Ltd, London, 1983.

59 S. Capaccioli, K. L. Ngai and N. Shinyashiki, J. Phys. Chem. B, 2007, 111, 8197-8209.

60 S. Murugavel and B. Roling, Phys. Rev. Lett., 2002, 89, 195902. 61 D. L. Sidebottom, Phys. Rev. Lett., 1999, 82, 3653-3656.

62 J. L. Green, J. Fan and C. A. Angell, J. Phys. Chem., 1994, 98, 13780-13790.
63 I. Iben, D. Braunstein, W. Doster, H. Frauenfelder, M. Hong, J. Johnson, S. Luck, P. Ormos, A. Schulte, P. Steinbach, A. Xie and R. Young, Phys. Rev. Lett., 1989, 62, 1916-1919.

64 F. Piazza, P. De Los Rios and Y.-H. Sanejouand, Phys. Rev. Lett., 2005, 94, 145502.

65 A. Xie, A. F. G. van der Meer and R. H. Austin, Phys. Rev. Lett., 2002, 88, 018102.

66 H. Shintani and H. Tanaka, Nat. Mater., 2008, 7, 870-877.

67 S. Khodadadi, A. Malkovskiy, A. Kisliuk and A. P. Sokolov, Biochim. Biophys. Acta, 2010, 1804, 15-19.

68 N. V. Surovtsev, Phys. Rev. E, 2001, 64, 061102.

69 A. A. R. Watt, J. P. Bothma and P. Meredith, Soft Matter, 2009, 5, 3754 .

70 M. D'Ischia, A. Napolitano, V. Ball, C. T. Chen and M. J. Buehler, Acc. Chem. Res., 2014, 47(12), 3541-3550.

71 G. W. Zajac, J. M. Gallas, J. Cheng, M. Eisner, S. C. Moss and A. E. Alvarado-Swaisgood, Biochim. Biophys. Acta, Gen. Subj., 1994, 1199, 271-278.

72 K. B. Stark, J. M. Gallas, G. W. Zajac, M. Eisner and J. T. Golab, J. Phys. Chem. B, 2003, 107, 11558-11562.

73 J. M. Gallas, K. C. Littrell, S. Seifert, G. W. Zajac and P. Thiyagarajan, Biophys. J., 1999, 77, 1135-1142.

74 C. A. Hunter and J. K. M. Sanders, J. Am. Chem. Soc., 1990, 112, 5525-5534.

75 L. K. Charkoudian and K. J. Franz, Inorg. Chem., 2006, 45, 3657-3664.

76 T. Miyake and M. Rolandi, J. Phys.: Condens. Matter, 2016, 28, 023001.

77 S. Khodadadi and A. P. Sokolov, Soft Matter, 2015, 11, 49844998.

78 S. Perticaroli, J. D. Nickels, G. Ehlers and A. P. Sokolov, Biophys. J., 2014, 106, 2667-2674.

79 R. Micillo, L. Panzella, K. Koike, G. Monfrecola, A. Napolitano and M. D'Ischia, Int. J. Mol. Sci., 2016, 17, 746. 